\title{
Mink SARS-CoV-2 infection in Poland - short communication
}

\author{
Katarzyna Domańska-Blicharz ${ }^{1 凶}$, Anna Orłowska², Marcin Smreczak², Krzysztof Niemczuk ${ }^{3}$, \\ Ewelina Iwan ${ }^{4}$, Arkadiusz Bomba ${ }^{4}$, Anna Lisowska ${ }^{1}$, Justyna Opolska ${ }^{1}$, Paweł Trębas ${ }^{2}$, \\ Patrycja Potyrało ${ }^{2}$, Magdalena Kawiak-Sadurska ${ }^{2}$, Jerzy Rola ${ }^{2}$ \\ ${ }^{1}$ Department of Poultry Diseases, ${ }^{2}$ Department of Virology, ${ }^{3}$ Director General, \\ ${ }^{4}$ Department of Omics Analyses, \\ National Veterinary Research Institute, 24-100 Puławy, Poland \\ domanska@piwet.pulawy.pl
}

Received: February 18, $2021 \quad$ Accepted: March 1, 2021

\begin{abstract}
Introduction: Since April 2020, when the first SARS-CoV-2 infection was reported in mink and subsequently in mink farm workers in the Netherlands, it has been confirmed that human-to-mink and mink-to-human transmission can occur. Later, SARS-CoV-2 infections in mink were reported in many European and North American countries. Material and methods: Samples from 590 mink from a total of 28 farms were tested by real-time RT-PCR. Whole genome sequences from one positive farm were generated and genetic relatedness was established. Results: SARS-CoV-2 RNA was detected on a breeder farm with stock of 5,850 mink. Active viraemia was confirmed in individually tested samples with $\mathrm{Ct}$ values respectively between 19.4 and 29.6 for $\mathrm{E}$ and $\mathrm{N}$ gene fragments. Further testing of samples from culled animals revealed $70 \%$ positivity in throat swabs and $30 \%$ seropositivity in blood samples. Phylogenetic analysis of full-length nucleotide sequences of two SARS-CoV-2 isolates revealed that they belong to the $20 \mathrm{~B}$ Nextstrain clade. Several nucleotide mutations were found in analysed samples compared to the reference Wuhan HU-1 strain and some of them were nonsynonymous. Conclusion: We report the infection of mink with SARS-CoV-2 on one farm in Poland and the results of subsequent analysis of virus sequences from two isolates. These data can be useful for assessment of the epidemiological situation of SARS-CoV-2 in Poland and how it endangers public health.
\end{abstract}

Keywords: SARS-CoV-2, WGS, Poland, mink.

\section{Introduction}

The first identified cases of Covid-19 (coronavirus disease 2019) in mink were detected in April 2020 in the Netherlands and later in other European countries including Spain, Denmark, Italy, Sweden, Greece, France and Lithuania $(15,19)$. In addition, infected mink have been detected in several states in the USA (Utah, Michigan, Oregon, and Wisconsin) and in Canada (19). In most of these cases, animals had an asymptomatic disease course, although there were sometimes observed mild respiratory symptoms and diarrhoea as well as pneumonia and increased mortality $(3,14,16,17,19)$. The source of severe acute respiratory syndrome coronavirus 2 (SARS-CoV-2) on infected mink farms has not been identified, but in a few cases in the Netherlands and Denmark it has been clearly demonstrated that the virus was transmitted from infected humans and then spread among mink $(9,15$, 16). However, the biggest threat was that SARS-CoV-2 would spill back from mink into the human population and that during the passage through mink the virus could generate mutations in its genome. Mutations in the gene coding the spike protein are particularly threatening because they are considered to jeopardize the potential efficacy of vaccination and antibody therapy (7). Preliminary studies by the Danish Statens Serum Institut indicated that SARS-CoV-2 Cluster 5 variant was less effectively neutralised by antibodies from humans infected with unmutated virus (10). The implemented biosecurity procedures on mink farms in most affected countries (especially in the Netherlands and Denmark) turned out to be insufficient, infections in mink have spread on a large scale, and the occurrence of SARS-CoV-2 in people connected to mink farms has also increased (3). Therefore, the European Centre for Disease Prevention 
and Control, World Health Organization and World Organisation for Animal Health (OIE) recommended close surveillance of mink farms $(5,18)$. In response to these recommendations, in the autumn of 2020 the Polish General Veterinary Inspectorate introduced official monitoring of mink farms. Additionally in December 2020, the Minister of Agriculture and Rural Development changed the law to recognise SARS-CoV-2 infection in mink as a notifiable disease (6). After the preventive elimination of mink farms in Denmark, Poland became the largest producer of mink pelts in Europe, although the number of active farms had decreased from 354 in 2020 to 266 at the beginning of 2021 (some farms have suspended or definitively terminated their activities).

\section{Material and Methods}

Sample collection and SARS-CoV-2 detection. During a period of three months between November 2020 and January 2021, 900 samples comprising 490 throat and 410 rectal swabs collected from 590 mink from 28 farms located in different regions of Poland were tested for SARS-CoV-2. After detection of the virus, additional samples from animals from one infected farm were tested. In total fifty such throat swabs and 150 serum samples from that farm's mink were tested. Swabs were suspended in phosphate buffered saline (Biomed, Poland), shaken and centrifuged for 10 minutes at $3000 \times \mathrm{g}$. Supernatant in a $140 \mu \mathrm{L}$ volume was used for RNA isolation with a QIAamp Viral RNA Mini Kit (Qiagen, Germany) following the manufacturer's protocol. A real-time reverse transcription polymerase chain reaction (rRT-PCR) was carried out as previously described for amplification of $113 \mathrm{bp}, 128 \mathrm{bp}$ and $100 \mathrm{bp}$ lengths of the E, N and both RdRp gene fragments respectively (4). The QuantiTect Probe RT-PCR Kit (Qiagen) was used for all rRT-PCR assays in an ABI 7500 or QuantStudio 6 instrument (Applied Biosystems, ThermoFisher Scientific, USA). An ID Screen SARS-CoV-2 double antigen multi-species ELISA commercial test (IDVet, France) was used for serological analysis of serum samples. To confirm the positive serological results obtained from ELISA, a cPass SARS-CoV-2 Neutralization Antibody Detection Kit (Nanjing GenScript Biotech, China) based on recombinant $\mathrm{S}$ protein fragment was used for virus neutralisation tests.

Virus sequencing and phylogenetic analysis. The attempt at obtaining the complete genome of the detected SARS-CoV-2 isolates was undertaken with short-read sequencing (Illumina, USA). Viral RNA extracted directly from positive throat swab samples was reverse transcribed into cDNA using a Superscript IV First-Strand cDNA Synthesis Kit (Invitrogen, USA). Then the second strand was synthesised with the Klenow polymerase (New England Biolabs, USA). The quantity and quality of dsDNA were verified with a Qubit 3.0 fluorometer (Thermo Fisher Scientific) and capillary electrophoresis with a 5200 Fragment Analyzer (Agilent, USA), respectively. Libraries were prepared using a Nextera XT sample preparation kit (Illumina). Pair-end sequencing $(2 \times 300 \mathrm{bp})$ was performed using a V3 MiSeq reagent kit (Illumina). Raw reads were trimmed with the use of fastp (https://academic.oup.com/ bioinformatics/article/34/17/i884/5093234) and viral sequences were extracted using BBDuk (sourceforge.net/ projects/bbmap/). Reads were mapped to original reference for SARS-CoV-2 (Wuhan-Hu-1: MN908947) by BWA MEM (12). Phylogenetic analysis was undertaken with the use of online tools: GISAID (ver. 14-02-2021, https://www.epicov.org) and NextClade (ver. 14-02-2021, https://nextstrain.org/).

\section{Results}

The swabs from 27 farms tested negative. However, in the examination of 20 swabs from one farm, 4 showed the presence of SARS-CoV-2-specific amplicons of all gene fragments (4) (Table 1).

Table 1. Results of SARS-CoV-2 testing for E, N and RdRp genes of individual throat swabs collected from infected mink

\begin{tabular}{ccccc}
\hline $\begin{array}{c}\text { Positive throat } \\
\text { swab }\end{array}$ & 3 & 8 & 11 & 12 \\
\hline E gene & 19.4 & 23.9 & 22.3 & 23.9 \\
N gene & 24.9 & 29.6 & 27.9 & 28.9 \\
RdRp1 & 29.9 & 37.8 & 34.3 & 36.2 \\
RdRp2 & 28.8 & 36.9 & 33.6 & 35.5 \\
\hline
\end{tabular}

The numbers indicate $\mathrm{Ct}$ values (defined as the number of cycles where the fluorescence generated by the presence of target sequence exceeds the background)

The infected farm was located in the Pomorskie voivodeship (in the north of Poland) and kept about 5,850 breeder minks which were healthy. After detection of SARS-CoV-2 and prior to culling and proper carcass disposal 120 blood samples were taken. Together with 20 throat swab samples from mink carcasses from this farm, they comprised sample set 1 . Throat swabs were also collected from 30 additional animals and blood samples were simultaneously taken from the same 30 animals, these being sample set 2 . In total 50 throat swabs and 150 serum samples were tested. The results are presented in Table 2.

Table 2. Summary of further sample testing from the infected mink

\begin{tabular}{ccccc}
\hline \multirow{2}{*}{ sample set } & \multicolumn{2}{c}{ throat swabs } & \multicolumn{2}{c}{ serum samples } \\
\cline { 2 - 5 } & studied & positive & studied & positive \\
\hline 1 & 20 & 20 & 120 & 30 \\
\hline 2 & 30 & 15 & 30 & 15 \\
\hline total & 50 & 35 & 150 & 45 \\
$(30 \%)$
\end{tabular}




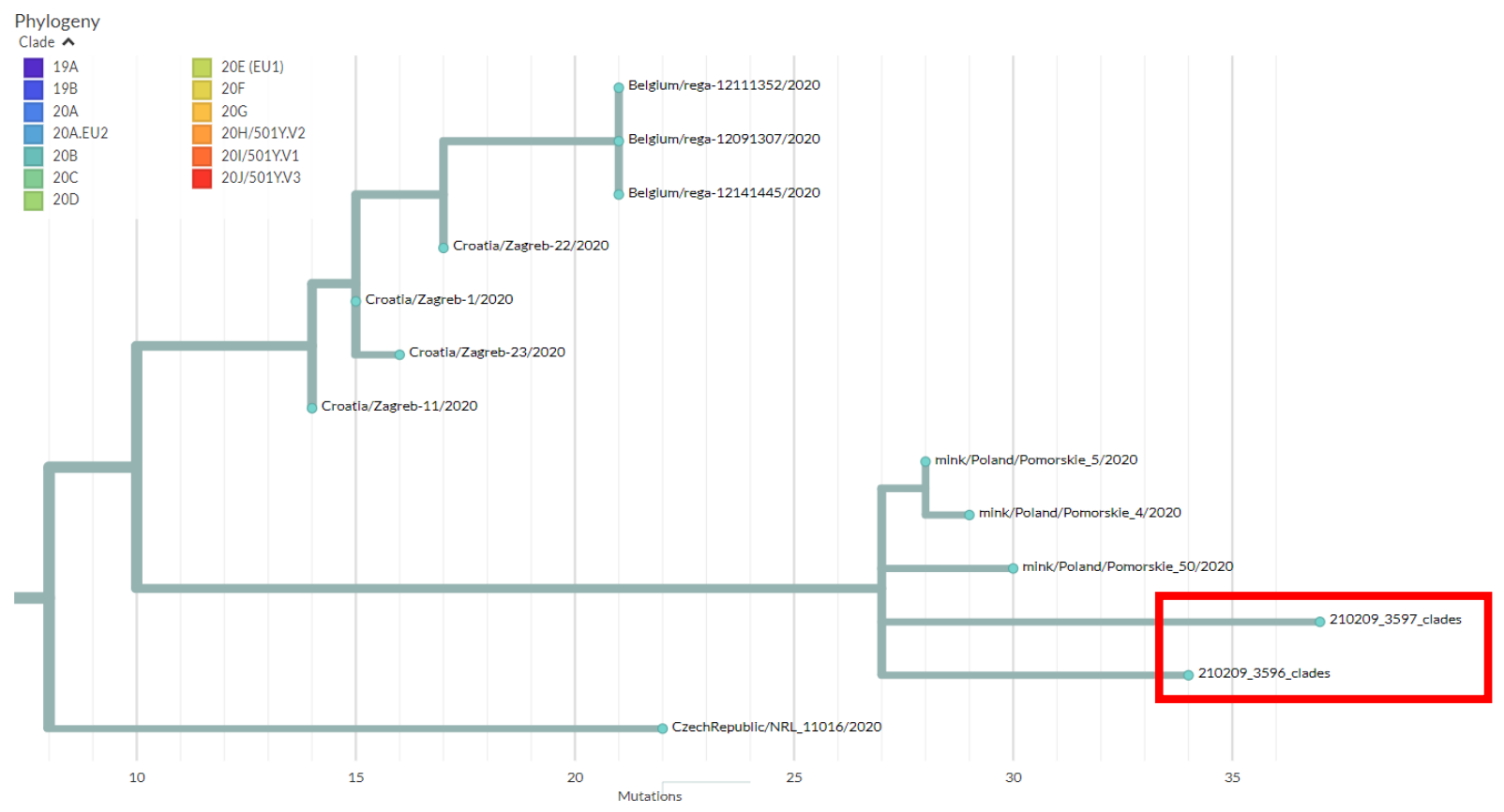

Fig. 1. Phylogenetic tree of SARS-CoV-2 sequenced in the present study. The sequences are available in the EpiCoV GISAID database. The SARS-CoV-2 isolates detected in mink in January 2021 are marked with the red box

Table 3. Summary of amino acid substitutions identified in two SARS$\mathrm{CoV}-2$ genomes detected in infected mink

\begin{tabular}{|c|c|c|}
\hline $\begin{array}{c}\text { SARS-CoV-2 } \\
\text { gene }\end{array}$ & $\begin{array}{c}\text { EPI_ISL_984307 } \\
\left(210209 \_3597 / 2021\right)\end{array}$ & $\begin{array}{c}\text { EPI_ISL_984305 } \\
\left(210209 \_3596 / 2021\right)\end{array}$ \\
\hline \multirow{5}{*}{$\mathrm{S}$} & $\mathrm{C} 1247 \mathrm{~F}$ & $\mathrm{C} 1247 \mathrm{~F}$ \\
\hline & D614G & D614G \\
\hline & G75V & G75V \\
\hline & Y453F & Y453F \\
\hline & - & M177T \\
\hline \multirow{3}{*}{$\mathrm{N}$} & D343Y & D343Y \\
\hline & G204L & G204L \\
\hline & R203K & R203K \\
\hline NS3 & R126M & - \\
\hline NS7b & L22stop & L22stop \\
\hline \multirow{3}{*}{ NSP2 } & $\mathrm{Q} 275 \mathrm{H}$ & Q275H \\
\hline & S138L & S138L \\
\hline & V541I & V541I \\
\hline \multirow{2}{*}{ NSP3 } & D218E & D218E \\
\hline & E869D & E869D \\
\hline \multirow{2}{*}{ NSP9 } & G37E & G37E \\
\hline & P57S & P57S \\
\hline NSP12 & P323L & P323L \\
\hline NSP13 & K94I & K94I \\
\hline \multirow{2}{*}{ NSP14 } & A $274 \mathrm{~S}$ & A $274 \mathrm{~S}$ \\
\hline & M49I & M49I \\
\hline \multirow[t]{2}{*}{ NSP15 } & M218T & M218T \\
\hline & Strain classification & \\
\hline $\begin{array}{l}\text { GISAID } \\
\text { clade }\end{array}$ & $\mathrm{O}$ & GR \\
\hline $\begin{array}{l}\text { PANGO } \\
\text { lineage }\end{array}$ & B.1.1.279 & B.1.1.279 \\
\hline $\begin{array}{l}\text { Nextstrain } \\
\text { clade }\end{array}$ & $20 \mathrm{~B}$ & $20 \mathrm{~B}$ \\
\hline
\end{tabular}

Viable numbers of mapped reads were obtained from two samples, 210209_3596 and 210209_3597, and were 10,103 and 12,848 , respectively. The respective average coverage extents of the SARS-CoV-2 genome were 9.1 (210209_3596) and 13.8 (210209_3597). Variant calling was carried out and consensus sequences were found using the BCTtools package (13). Two fulllength SARS-CoV-2 genomes were obtained from throat swabs nos 11 and 12 (Table 1) and consisted of 29,891 nucleotides each. They were submitted to the GISAID EpiCoV database with the strain IDs EPI_ISL_984305 and EPI_ISL_984307. Phylogenetic analysis indicated that these viruses belong to the 20B Nextstrain clade (Fig. 1).

According to GISAID classification, both strains were of B.1.1.279 lineage, although of two different clades, $\mathrm{O}$ and GR. This discrepancy probably results from relatively low sequence coverage in one of the regions used by the GISAID tool for virus classification. In addition, many nucleotide mutations were found compared to the genome sequence of the reference strain and some of them were nonsynonymous (Table 3).

\section{Discussion}

SARS-CoV-2 was identified as an emerging coronavirus in humans in December 2019. It is now well documented that the SARS-CoV-2 virus can be transmitted between humans and animals and subsequently circulate in infected animals and be transmitted back to humans, posing a risk to public health $(9,15,16)$. Like the ferret, the mink, belongs to the Mustelidae family and is highly susceptible to SARS-CoV-2 infection due to the fact that they have similarities to humans in lung physiology, 
cellular receptor distribution and clinical signs of infection. The scale of infections in farmed mink which is observed just over a year after the outbreak of the pandemic is worrying, mainly due to the unlimited possibility of the virus to pass through a sometimes large population of highly susceptible animals which potentially enables it to acquire new traits, including changed antigenicity and virulence. Moreover, any escape of such animals from a farm can create a SARS-CoV-2 reservoir in wildlife and as shown by the example of highly pathogenic avian influenza, measures to minimize or eliminate such a reservoir practically doomed to failure.

In the spike protein of SARS-CoV-2 detected on a Polish mink farm, four or five amino acid substitutions were identified, depending on the isolate. Among the identified changes, only one substitution of tyrosine to phenylalanine (Y453F) is located in the receptorbinding domain (8). This location is not regarded as critical for binding to ACE2 receptors but it is described as responsible for increased binding affinity to cellular receptors in mink $(1,11)$. This substitution was detected in people who had no contact with mink and even in countries not connected with the mink industry, such as South Africa, the Faroe Islands, Norway and Switzerland. It also appears that this change was present in SARS-CoV-2-infected Danes several months earlier than it was detected in minks. So far it has not been identified in people in Poland (GISAID database: https://platform.gisaid.org/epi3/). The Y453F S substitution and two others, del $69 \mathrm{H}$ and $70 \mathrm{~V}$, which were additionally described in mink in the Cluster 5 variant of SARS-CoV-2, were recently detected in a lymphoma patient in Russia (2). It is suggested that these changes were probably favoured by selection pressure, which is common to immunocompromised patients. There is a high risk that such changed virus variants can later spill over into the human population. So it seems that the Y453F substitution should not be treated as minkassociated. It should also be emphasised that SARS-CoV-2 isolates identified on a Polish mink farm do not belong to any of the "variants of concern" reported so far.

In summary, in this report we confirmed the infection of mink with SARS-CoV-2 on a farm in Poland and presented the subsequent analysis of virus sequences from two isolates. These data can be useful for future assessments of the epidemiological situation of SARS$\mathrm{CoV}-2$ in Poland to maintain vigilance for public health.

Conflict of Interests Statement: The authors declare that there is no conflict of interests regarding the publication of this article.

Financial Disclosure Statement: This study was covered by resources of the National Veterinary Research Institute, Puławy, Poland.

Animal Rights Statement: This article's authors did not initiate any of the procedures on animals. Sampling was carried out by veterinary officers, who took samples in the framework of regulatory veterinary inspection and under the permission of the farm owner. For these reasons, sampling did not require the approval of the Ethics Committee.

\section{References}

1. Andersen K.G., Rambaut A., Lipkin W.I., Holmes E.C., Garry R.F.: The proximal origin of SARS-CoV-2. Nat Med 2020, 26, 450-452, doi: 10.1038/s41591-020-0820-9.

2. Bazykin G.A., Stanevich O., Danilenko D., Fadeev A., Komissarova K., Ivanova A., Sergeeva M., Safina K., Nabieva E., Klink G., Garushyants S., Zabutova J., Kholodnaia A., Skorokhod I., Ryabchikova V.V., Komissarov A., Lioznov D.: Emergence of Y453F and $\triangle 69-70 \mathrm{HV}$ mutations in a lymphoma patient with long-term COVID-19. Virological.org. https:/virological.org/t/ emergence-of-y453f-and-69-70hv-mutations-in-a-lymphoma-patientwith-long-term-covid-19/580.

3. Boklund A., Hammer A.S., Quaade M.L., Rasmussen T.B., Lohse L., Strandbygaard B., Jørgensen C.S., Olesen A.S., Hjerpe F.B., Petersen H.H., Jensen T.K., Mortensen S., Calvo-Artavia F.F., Lefèvre S.K., Nielsen S.S., Halasa T., Belsham G.J., Bøtner A.: SARS-CoV-2 in Danish Mink Farms: Course of the Epidemic and a Descriptive Analysis of the Outbreaks in 2020. Animals 2021, 11, 164, doi: 10.3390/ani11010164.

4. Corman V.M., Landt O., Kaiser M., Molenkamp R., Meijer A., Chu D.K., Bleicker T., Brünink S., Schneider J., Schmidt M.L., Mulders D.G.J.C., Haagmans B.L., van der Veer B., van den Brink S., Wijsman L., Goderski G., Romette J.-L., Ellis J., Zambon M., Peiris M., Goossens H., Reusken C., Koopmans M.P.G., Drosten C.: Detection of 2019 novel coronavirus (2019-nCoV) by real-time RT-PCR. Euro Surveill 2020, 25, 2000045, doi: 10.2807/1560-7917. ES.2020.25.3.200004.

5. European Centre for Disease Prevention and Control: Detection of new SARS-CoV-2 variants related to mink. Rapid risk assessment 12 Nov 2020. https://www.ecdc.europa.eu/sites/default/files/ documents/RRA-SARS-CoV-2-in-mink-12-nov-2020.pdf.

6. Government of the Republic of Poland: Rozporządzenie Ministra Rolnictwa i Rozwoju Wsi z dnia 15 grudnia 2020 r. w sprawie zwalczania zakażenia SARS-CoV-2 u norek. Dz U 2020, 264, poz. 2302. (Official Journal of Laws 2020, 264, item 2302).

7. Koopmans M.P.G.: SARS-CoV-2 and the human-animal interface: outbreaks on mink farms. Lancet Infect Dis 2021, 21, 18-19, doi.org/10.1016/S1473-3099(20)30912-9.

8. Lan J., Ge J., Yu J., Shan S., Zhou H., Fan S., Zhang Q., Shi X., Wang Q., Zhang L., Wang X.: Structure of the SARS-CoV-2 spike receptor-binding domain bound to the ACE2 receptor. Nature 2020, 581, 215-220, doi: 10.1038/s41586-020-2180-5.

9. Larsen H.D., Fonager J., Lomholt F.K., Dalby T., Benedetti G., Kristensen B., Urth T.R., Rasmussen M., Lassaunière R., Rasmussen T.B., Strandbygaard B., Lohse L., Chaine M., Møller K.L., Berthelsen A.N., Nørgaard S.K., Sönksen U.W., Boklund A.E., Hammer A.S., Belsham G.J., Krause T.G., Mortensen S., Bøtner A., Fomsgaard A., Mølbak K.: Preliminary report of an outbreak of SARS-CoV-2 in mink and mink farmers associated with community spread, Denmark, June to November 2020. Euro Surveill 2021, 26, 2100009, doi: 10.2807/15607917.ES.2021.26.5.210009.

10. Lassaunière R., Fonager J., Rasmussen M., Frische A., Polacek Strandh C., Rasmussen B.T., Bøtner A., Fomsgaard A.: SARSCoV-2 spike mutations arising in Danish mink and their spread to humans. Statens Serum Institut, Copenhagen, 2020. https://files.ssi.dk/Mink-cluster-5-short-report_AFO2.

11. Lauring A.S., Hodcroft E.B.: Genetic Variants of SARS-CoV-2What Do They Mean? JAMA 2021, 325, 529-531, doi:10.1001/jama.2020.27124. 
12. Li H.: Aligning sequence reads, clone sequences and assembly contigs with BWA-MEM. arxiv 2013. arXiv:1303.3997v2 (q-bio.GN).

13. Li H., Handsaker B., Wysoker A., Fennell T., Ruan J., Homer N., Marth G., Abecasis G., Durbin R., 1000 Genome Project Data Processing Subgroup: The Sequence Alignment/Map format and SAMtools. Bioinformatics 2009, 25, 2078-2079, doi: 10.1093/bioinformatics/btp352.

14. Molenaar R.J., Vreman S., Hakze-van der Honing R.W., Zwart R., de Rond J., Weesendorp E., Smit L.A.M., Koopmans M.P.G., Bouwstra R.J., Stegeman A., van der Poel W.H.M.: Clinical and Pathological Findings in SARS-CoV-2 Disease Outbreaks in Farmed Mink (Neovison vison). Vet Pathol 2020, 57, 653-657, doi: 10.1177/0300985820943535.

15. Oreshkova N., Molenaar R.J., Vreman S., Harders F., Oude Munnink B.B., Hakze-van der Honing R.W., Gerhards N., Tolsma P., Bouwstra R.J., Sikkema R.S., Tacken M.G., de Rooij M.M., Weesendorp E., Engelsma M.Y., Bruschke C.J., Smit L.A.M., Koopmans M.P.G., van der Poel W.H.M., Stegeman A.: SARSCoV-2 infection in farmed minks, the Netherlands, April and May 2020. Euro Surveill 2020, 25, 2001005, doi: 10.2807/15607917.ES.2020.25.23.2001005.

16. Oude Munnink B.B., Sikkema R.S., Nieuwenhuijse D.F., Molenaar R.J., Munger E., Molenkamp R., van der Spek A.,
Tolsma P., Rietveld A., Brouwer M., Bouwmeester-Vincken N., Harders F., Hakze-van der Honing R.W., Wegdam-Blans M.C.A., Bouwstra R.J., GeurtsvanKessel C., van der Eijk A.A., Velkers F.C., Smit L.A.M., Stegeman A., van der Poel W.H.M., Koopmans M.P.G.: Transmission of SARS-CoV-2 on mink farms between humans and mink and back to humans. Science 2021, 371, 172-177, doi: 10.1126/science.abe5901.

17. Sharun K., Tiwari R., Natesan S., Dhama K.: SARS-CoV-2 infection in farmed minks, associated zoonotic concerns, and importance of the One Health approach during the ongoing COVID-19 pandemic. Vet Q 2021, 41, 50-60, doi: 10.1080/01652176.2020.1867776.

18. World Health Organization: Disease outbreak news, 6 November 2020: SARS-CoV-2 mink-associated variant strain - Denmark. https://www.who.int/csr/don/06-november-2020-mink-associatedsarscov2-denmark/en.

19. World Organisation for Animal Health (OIE): Covid-19 portal: Events in animals 15 February 2021 update. https://www.oie.int/scientific-expertise/specific-information-andrecommendations/questions-and-answers-on-2019novel-coronavirus/ events-in-animals/. 